\title{
Maryvonne Prévot, Catholicisme social et urbanisme. Maurice Ducreux (1924-1958) et la fabrique de la Cité
}

Rennes, Presses universitaires de Rennes, coll. « Histoire », 2015, 480 p.

\section{Olivier Chatelan}

\section{OpenEdition}

Journals

Édition électronique

URL : http://journals.openedition.org/assr/27435

DOI : 10.4000 /assr.27435

ISSN : $1777-5825$

Éditeur

Éditions de l'EHESS

Édition imprimée

Date de publication : 1 octobre 2015

Pagination : 351

ISBN : 978-2-7132-2515-4

ISSN : 0335-5985

\section{Référence électronique}

Olivier Chatelan, «Maryvonne Prévot, Catholicisme social et urbanisme. Maurice Ducreux (1924-1958) et la fabrique de la Cité », Archives de sciences sociales des religions [En ligne], 172 I octobre-décembre, mis en ligne le 08 juin 2016, consulté le 24 septembre 2020. URL : http://journals.openedition.org/ assr/27435; DOI : https://doi.org/10.4000/assr.27435 


\section{Maryvonne Prévot, Catholicisme social et urbanisme. Maurice Ducreux (1924-1958) et la fabrique de la Cité}

Rennes, Presses universitaires de Rennes, coll. « Histoire », 2015, 480 p.

Olivier Chatelan

\section{RÉFÉRENCE}

Maryvonne Prévot,Catholicisme social et urbanisme. Maurice Ducreux (1924-1958) et la fabrique de la Cité, Rennes, Presses universitaires de Rennes, coll. « Histoire », 2015, $480 \mathrm{p}$. 
1 L'ouvrage que publie Maryvonne Prévot, maître de conférences en aménagement et urbanisme, intéresse directement les historiens, sociologues et politistes spécialistes du champ religieux. Il est le fruit d'une habilitation à diriger des recherches appuyée sur un travail de longue haleine qui revendique une pluridisciplinarité dans l'interprétation des résultats. L'étude se propose d'analyser les interférences multiples entre "références confessionnelles" et «références savantes» dans le petit

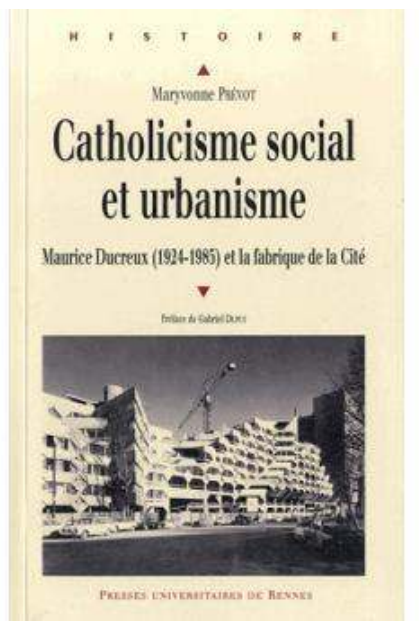
monde des organismes d'étude tournés vers l'urbain en France au cours des années 1960-1980. L'hypothèse de départ est la suivante: la pléiade des structures de conseil et d'expertise en urbanisme et aménagement qui naissent et se reconfigurent constamment au cours de la période serait marquée, dans son personnel, par la surreprésentation ou tout au moins par la présence significative de militants "d'origine chrétienne ». Pour tester la validité de cette hypothèse, M. Prévot s'est intéressée à la trajectoire d'un prêtre incardiné à la Mission de France, Maurice Ducreux (1924-1985), dont les archives privées - en particulier des notes personnelles - ont pu être dépouillées.

Les étapes de la vie religieuse et surtout professionnelle de Ducreux fournissent le plan du livre qui s'ordonne autour de cinq moments. Le chapitre 1 évoque logiquement les premiers choix et les premiers engagements du jeune Maurice né à Paris : études au Petit séminaire de la Maladrerie à Caen dans lequel il anime une section de la Jeunesse étudiante chrétienne (JEC) ; entrée en 1944 au séminaire de la Mission de France qui s'est ouvert deux ans plus tôt à Lisieux ; immersion à partir de 1946 dans le milieu communiste d'Ivry-sur-Seine où il se lie d'amitié avec des chrétiens progressistes héritiers et contemporains de la pensée de Madeleine Delbrêl. Ordonné par le cardinal Feltin en juin 1950 pour le diocèse de Bayeux (il sera incardiné à la Mission de France en 1955), il occupe un emploi à temps partiel au Bureau municipal de la petite enfance à Ivry, qui le conduit notamment à assurer pendant trois ans l'animation de la colonie des Mathes, en Charente-Maritime, où il contribue au succès de la "République de Villanous ", une utopie urbaine forgée dans le jeu sur un modèle proche de la pédagogie scoute. Ce premier moment, qui est aussi celui de la proximité avec le mouvement Jeunesse de l'Église de Maurice Montuclard, marque la politisation de Ducreux au contact du PCF, dans un compagnonnage actif et assumé.

3 Le deuxième chapitre analyse l'entrée de Maurice Ducreux dans le Bureau d'études et de réalisations urbaines (BERU), qui est aussi son entrée « en » urbanisme. Confronté à la crise du logement dans les paroisses de la banlieue parisienne (Alfortville, Colombes, Gennevilliers) où il est successivement envoyé au début des années 1950, il se rapproche du mouvement d'autoconstruction castor, avant de participer aux premiers pas du BERU créé en 1957 autour de Max et Irène Stern. Là, dans cet organisme privé bien ancré dans le marché florissant des études d'aménagement et d'assistance à la maîtrise d'ouvrage, il se forme sur le terrain comme la plupart des membres, largement autodidactes. Fascinés par la sociologie urbaine naissante qui fournit l'armature 
conceptuelle et apolitique d'une planification technocratique, Ducreux et ses amis contribuent donc directement à la capitalisation de compétences techniques au sein du BERU, lequel joue un rôle de premier plan dans la rénovation du centre ancien d'Ivry, véritable laboratoire du logement collectif sous municipalité communiste.

Dans le troisième chapitre sont décrites les étapes de la consolidation et de la diversification des travaux du BERU, en Basse-Seine puis à l'étranger. Kinshasa en particulier fait l'objet d'une enquête sociodémographique en 1967-1969, période qui coïncide avec l'envoi de prêtres de la Mission de France dans la capitale congolaise. Maurice Ducreux fait partie de l'équipe du BERU sur place et travaille avec le jésuite belge Léon de Saint Moulin pour le compte de la Mission française d'urbanisme. Le chapitre suivant s'intéresse à la "crise du langage religieux " chez Ducreux au cours des années 1970. Le prêtre prend sa carte au PCF en 1973 et participe au lancement de la revue de sociologie d'inspiration marxiste Espaces et sociétés. Cette même année, il quitte le BERU pour devenir chercheur contractuel au sein de l'Unité de recherche appliquée de l'École spéciale d'architecture à Paris, mais la précarité de son embauche empoisonne les relations avec son employeur. Le cinquième et dernier chapitre décrit d'abord son activité d'expert au PCF comme spécialiste reconnu des questions urbaines, puis ses prises de distance avec le parti qu'il quitte en 1977, avant un ultime engagement au sein du Mouvement contre le racisme et pour l'amitié entre les peuples (MRAP) au cours des quatre dernières années de sa vie. L'ensemble est complété par un index très utile, un dossier photographique et une annexe appréciable sur le parcours de plusieurs des acteurs évoqués au fil des pages.

5 Cette étude constitue une très belle contribution à la compréhension des relations entre engagement catholique et fabrique de la ville et de l'urbain en France au $\mathrm{xx}^{\mathrm{e}}$ siècle. Le travail est bien documenté : l'auteur a croisé les papiers Ducreux avec plusieurs fonds d'archives publiques et privées (archives municipales d'Ivry-sur-Seine, CFDT, Mission de France, Institut français d'architecture) auxquels elle a confronté le résultat d'une série d'entretiens. La masse d'informations collectées a permis une vaste entreprise de mise en réseau des dizaines d'acteurs identifiés : chaque militant ou personnalité proche de Ducreux - ou susceptible d'avoir eu une influence sur son parcours, intellectuel comme professionnel - fait l'objet d'un ou de plusieurs paragraphes biographiques qui resituent le personnage à la fois dans un réseau d'interconnaissances et dans une mouvance idéologique. De ce point de vue, ce travail d'archéologie des pratiques professionnelles de l'urbanisme est remarquable : l'auteur excelle - et manifestement se passionne - à identifier des connexions, des passeurs ou des livres qui donnent sens à des engagements. Par ailleurs, l'ouvrage propose des pistes d'interprétation souvent très pertinentes pour mettre au jour les fils très enchevêtrés (et parfois tus) entre socialisation religieuse, professionnalisation et militantisme laïc. Les conflits intérieurs sur la double appartenance (à l'Église et aux organismes de recherche) ou les allers-retours sur ce que signifie la fidélité à soi et aux autres ne sont pas niés mais constituent au contraire le fil directeur de l'ouvrage : il y a dans ce livre la démonstration qu'une histoire religieuse seulement tournée vers le profil confessionnel des acteurs méconnait une partie essentielle de la complexité des trajectoires individuelles ou collectives et peut s'avérer insuffisante, dès lors, pour expliquer des choix décisifs dans la construction d'un parcours. Enfin, il faut saluer le remarquable balisage bibliographique qui scande régulièrement l'étude. Un nombre considérable d'auteurs, historiens mais également philosophes, sociologues, politistes ou géographes, sont mobilisés explicitement pour éclairer telle ou telle difficulté 
d'analyse ou d'interprétation, même si l'on peut regretter que le très bon livre d'Éric Le Breton sur l'histoire de la sociologie urbaine française (PUR, 2012) ait été d'abord cité pour ses seuls manques (sur Chombart de Lauwe et Uriage). L'auteur engage la discussion pied à pied avec ces auteurs, dans une confrontation critique stimulante.

On évoquera toutefois ici deux regrets, qui portent sur la conception générale de l'étude. En premier lieu, la grande érudition de l'auteur sur son sujet la conduit, on l'a dit, à multiplier les références sur les réseaux qui enserrent les hommes et les femmes dont il est question à chaque page du livre. Cette abondance d'informations devient parfois vertige car on perd le fil de la démonstration. Si l'intention est parfaitement louable et bienvenue de décrire ce qui se joue et se noue au raz des équipes et des rencontres, sa transposition sous la forme de notices biographiques ou de mises au point théoriques, insérées au fil de la démonstration, nuit à la compréhension d'ensemble. Dès lors, le parcours de Ducreux apparaît seulement en filigrane et le lecteur peine à en rassembler les principales étapes. Il est vrai cependant que les titres de sous-parties y remédient au moins partiellement.

7 La seconde critique porte sur le projet : l'auteur utilise la trajectoire et le récit sur soi de Ducreux pour penser plus largement l'articulation entre appartenance confessionnelle et engagement dans la recherche technique et scientifique. Il n'y a pas là d'incompatibilité a priori sur le plan méthodologique et on a salué plus haut l'essai à part entière que constitue le livre. Il reste que le risque est d'attribuer à la trajectoire de Ducreux des analyses qui correspondent parfois à des situations analogues mais qui restent extérieures à son parcours. Par exemple, l'auteur consacre de longs développements (p. 64-69) à des expériences situées d'habitat communautaire (le « 14 rue de Sèvres ", l'apport-travail des castors, le rôle de personnalités comme Gilbert Gadoffre, Roger Lavialle ou André Depierre) qui n'ont pu avoir qu'une influence très indirecte sur les choix de Ducreux. La contextualisation remarquable effectuée par l'auteur ne signifie pas qu'il y ait eu influences réciproques: l'administration de la preuve pose problème. Il faut reconnaître toutefois qu'il s'agit là d'une difficulté propre à tous les chercheurs qui prennent pour terrain des sphères sécantes de militance : les interférences nombreuses entre des appartenances multiples et concomitantes chez ces "catholiques de gauche» rendent poreuse - et donc incommode à évaluer - la frontière parfois ténue entre engagements dans le siècle et engagements religieux. 